\title{
The Role of Glucose Limitation in the Regulation of the Transport of Glucose, Gluconate and 2-Oxogluconate, and of Glucose Metabolism in Pseudomonas aeruginosa
}

\author{
By P. H. WHITING*, M. MIDGLEY AND E.A. DAWES \\ Department of Biochemistry, University of Hull, Hull HU6 ${ }_{7} R X$
}

(Received I September 1975)

\begin{abstract}
SUMMARY
The pathway of glucose metabolism in Pseudomonas aeruginosa was regulated by the availability of glucose and related compounds. On changing from an ammonium limitation to a glucose limitation, the organism responded by adjusting its metabolism substantially from the extracellular direct oxidative pathway to the intracellular phosphorylative route. This change was achieved by repression of the transport systems for gluconate and 2-oxogluconate and of the associated enzymes for 2-oxogluconate metabolism and gluconate kinase, while increasing the levels of glucose transport, hexokinase and glucose 6-phosphate dehydrogenase. The role of gluconate, produced by the action of glucose dehydrogenase, as a major inhibitory factor for glucose transport, and the possible significance of these regulatory mechanisms to the organism in its natural environment, are discussed.
\end{abstract}

\section{INTRODUCTION}

Glucose metabolism in Pseudomonas aeruginosa is complex (Fig. I), involving both direct oxidative and phosphorylative routes of metabolism (Stokes \& Campbell, 195I; Claridge \& Werkman, 1953, 1954; Wang, Stern \& Gilmour, 1959; Roberts, Midgley \& Dawes, 1973). This apparent complexity of metabolism has been clarified by the discovery that these two routes occur in different compartments of the cell. Thus, the enzymes of the direct oxidative pathway, glucose dehydrogenase and gluconate dehydrogenase, are oriented in the cytoplasmic membrane in such a manner that they oxidize their substrates extracellularly, i.e. in the periplasmic space (Midgley \& Dawes, 1973; Roberts et al., 1973). The products of their activity, gluconate and 2-oxogluconate respectively, are taken up by specific transport systems before their intracellular phosphorylation (Fig. 2). 2-Oxogluconate 6-phosphate is then reduced to gluconate 6-phosphate (Roberts et al., I973). Glucose, which is taken up by a separate transport system of rather broad specificity (Midgley \& Dawes, I973), is phosphorylated and oxidized to gluconate 6-phosphate, so that the metabolism of glucose, gluconate and 2-oxogluconate converges on gluconate 6-phosphate, which is further metabolized principally via the Entner-Doudoroff route but also by the pentose phosphate pathway (Wang et al., 1959).

Previously we have studied the regulation of the enzymes of glucose catabolism by citrate under conditions of nitrogen-limitation in a chemostat (Hamlin, $\mathrm{Ng} \&$ Dawes, $1967 ; \mathrm{Ng} \&$ Dawes, 1973) and more recently this work has been extended to include also the regulation of the transport systems for glucose, gluconate and 2-oxogluconate (Whiting, Midgley \&

\footnotetext{
* Present address: Department of Biochemistry, University of Nottingham.
} 


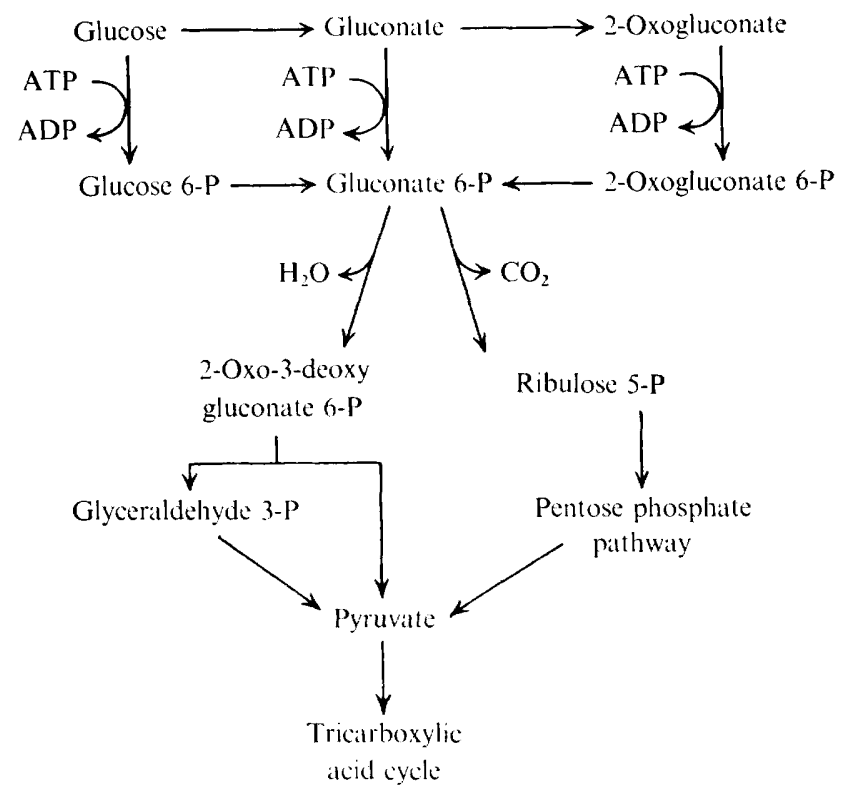

Fig. I. Pathways of glucose metabolism in $P$. aeruginosa.

Dawes, 1976). Conditions of nitrogen limitation were chosen, to enhance catabolite repression by carbon compounds.

In its natural environment, however, $P$. aeruginosa will also encounter conditions of carbon limitation and we have therefore compared the effects of glucose limitation with nitrogen limitation on the transport systems for glucose, gluconate and 2-oxogluconate, and on enzymes characteristic of the extracellular and intracellular pathways of glucose metabolism. Evidence is presented which reveals that $P$. aeruginosa responds to a glucose limitation by switching its metabolism of glucose substantially from the extracellular direct oxidative pathway to the intracellular phosphorylative route. A preliminary account of these findings has been presented by Dawes, Midgley \& Whiting (1976).

\section{Periplasmic space}

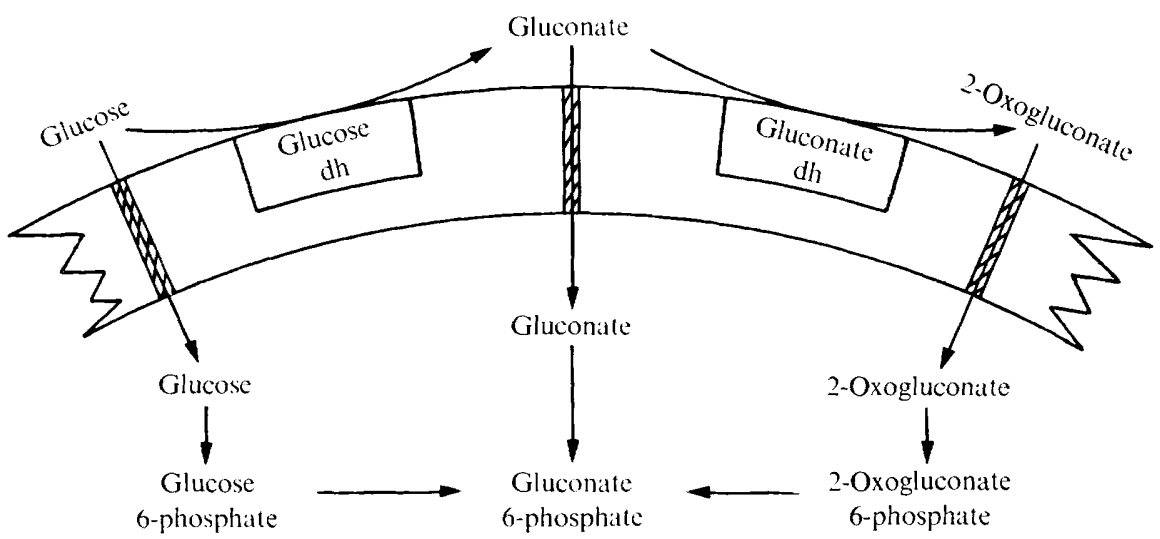

Fig. 2. The extracellular and intracellular pathways of glucose metabolism in P. aeriginosa. dh, Dehydrogenase. 


\section{METHODS}

Organism. The organism used was Pseudomonas aeruginosa PAOI, kindly provided by Professor B. W. Holloway.

Growth and maintenance of cultures. Routine maintenance, batch growth of the organism, harvesting procedures and the preparation of bacterial extracts were as previously described (Ng \& Dawes, I973; Midgley \& Dawes, 1973). For chemostat inocula the organism was subcultured at least ten times in the appropriate medium.

The chemostat was identical to that used by $\mathrm{Ng} \&$ Dawes (1973), the dilution rate being $0.4 \mathrm{I} \mathrm{h}^{-1}$ throughout. Medium for continuous cultivation was prepared in 201 batches which were sterilized by filtration through a Sartorius filter (I $42 \mathrm{~mm}$ diam, pore size $0.22 \mu \mathrm{m}$ ) at $137 \cdot 5 \mathrm{kN} / \mathrm{m}^{2}\left(20 \mathrm{lb} / \mathrm{in}^{2}\right)$. The medium contained $(\mathrm{g} / 20 \mathrm{l}): \mathrm{KH}_{2} \mathrm{PO}_{4}, \mathrm{IO} 8 ;\left(\mathrm{NH}_{4}\right)_{2} \mathrm{SO}_{4}, 3 \mathrm{I} \cdot 7$ (for nitrogen-limited growth) or 63.4 (for glucose-limited growth); nitrilotriacetic acid, $5 \cdot 73$; trace metals solutions I (I00 ml), 2 (105 ml) and 3 (2 ml) ( Ng \& Dawes, 1973); glucose, I 62.2 (for nitrogen-limited growth) or 108 (for glucose-limited growth).

Transport studies. These were performed as described by Midgley \& Dawes (1973). Organisms were separated from the suspending medium by rapid filtration and subsequently washed at $18{ }^{\circ} \mathrm{C}$ with a solution of the following composition : $4 \mathrm{~mm}-\mathrm{MgSO}_{4}, 0.342 \mathrm{M}-\mathrm{NaCl}$ in $0.067 \mathrm{M}$-potassium phosphate buffer adjusted to $\mathrm{pH} 7 \cdot \mathrm{I}$ with $\mathrm{IO} \mathrm{M}-\mathrm{NaOH}$.

The specific activities and final concentrations of the substrates used were: glucose, I00 $\mu \mathrm{Ci} / \mu \mathrm{mol}, 5 \mu \mathrm{m}$; sodium gluconate, $3.4 \mu \mathrm{Ci} / \mu \mathrm{mol}$, $50 \mu \mathrm{M}$; sodium 2-oxogluconate, $0 \cdot 1 \mu \mathrm{Ci} / \mu \mathrm{mol}, 3 \mathrm{~mm}$. The bacterial density was adjusted so that linear initial rates were measured in all assays. The radioactivity accumulated by the organism was determined as described by Midgley \& Dawes (1973).

Enzyme assays. The enzymes of glucose and 2-oxogluconate catabolism were assayed under the conditions described by $\mathrm{Ng} \&$ Dawes (1973) except that glucose dehydrogenase was assayed in the presence of phenazine methosulphate $(2 \mathrm{~mm})$. The combined activity of 2-oxogluconate kinase and 2-oxogluconate 6-phosphate reductase was assayed in the system: tris- $\mathrm{HCl}$ buffer (0.I $2 \mathrm{M}, \mathrm{pH} 7.5), \mathrm{I} \cdot 0 \mathrm{ml} ; \mathrm{MgCl}_{2}$ (0.6 M), O.I ml; NADPH (2 mM), O.I ml; ATP ( $36 \mathrm{~mm}, \mathrm{pH} 7.5), 0.3 \mathrm{ml}$; sodium 2-oxogluconate $(50 \mathrm{~mm}), 0 . \mathrm{I} \mathrm{ml}$; and distilled water to $2.8 \mathrm{ml}$. The reaction was monitored by following the ATP- and 2-oxogluconate-dependent oxidation of NADPH at $340 \mathrm{~nm}$ after addition of $0.1 \mathrm{ml}$ bacterial extract.

Preparation of $2-o x o\left[U-{ }^{14} C\right] g l u c o n a t e$. This method was based on the observations of Stokes \& Campbell (I951) who showed that dried cells of $P$. aeruginosa converted glucose quantitatively to 2-oxogluconate. A suspension of dried $P$. aeruginosa PAOI was prepared from an overnight culture grown in nutrient broth plus $\mathrm{I} \%(\mathrm{w} / \mathrm{v})$ glucose. Bacteria were harvested, washed and finally resuspended in distilled water. The suspension was dried by rotary evaporation at $40^{\circ} \mathrm{C}$ under reduced pressure. The dried organism was powdered and stored at $4{ }^{\circ} \mathrm{C}$. The conversion of $\left[\mathrm{U}^{14} \mathrm{C}\right]$ glucose to 2 -oxogluconate was performed in a Warburg manometer to monitor the extent of the reaction. Manometer flasks contained, in the main compartment, $0.5 \mathrm{ml}$ bacterial suspension (at $40 \mathrm{mg}$ dried organism $/ \mathrm{ml}$ distilled water), and $1.5 \mathrm{ml}$ potassium phosphate buffer $\left(0.067 \mathrm{M}, \mathrm{pH}\right.$ adjusted to 7.1 with $10 \mathrm{M}^{-}$ $\mathrm{NaOH})$. The side arm contained either glucose $(5 \mu \mathrm{mol}, 200 \mu \mathrm{Ci})$ or, for endogenous controls, water $(0.5 \mathrm{ml})$. The centre well contained a roll of Whatman No. 54I filter paper and $0.2 \mathrm{ml}$ of $20 \%(\mathrm{w} / \mathrm{v}) \mathrm{KOH}$. After equilibration and mixing of the flask contents, oxygen uptake was followed for $2 \mathrm{~h}$, after which the endogenous and reaction rates coincided. The flask contents were then centrifuged $(5000 \mathrm{~g}, 10 \mathrm{~min})$, and the supernatant was retained and the pellet washed with distilled water. The wash fluid and the original supernatant were 
Table I. The activities of the transport systems for glucose, gluconate and 2-oxogluconate in $P$. aeruginosa PAOI grown on nitrogen- and glucose-limited media

\begin{tabular}{lccc} 
& \multicolumn{2}{c}{ Rate of transport* $(\mu \mathrm{mol} / \mathrm{min} / \mathrm{g}$ dry $w \mathrm{t})$} \\
Limiting & Glucose & Gluconate & 2-Oxogluconate \\
nutrient & 2.9 & 19.7 & 47.9 \\
Ammonium & 2.7 & 22.4 & 55.8 \\
& 15.1 & 2.9 & 9.6 \\
Glucose & 14.7 & 3.4 & 11.4
\end{tabular}

* The two values quoted are the means of duplicate determinations performed on two separate culture samples from each steady state.

combined and treated at $60{ }^{\circ} \mathrm{C}$ with ethanol (final concentration $70 \%, \mathrm{v} / \mathrm{v}$ ). The protein precipitate was removed by filtration, and the filtrate rotary-evaporated to dryness at $40{ }^{\circ} \mathrm{C}$ under reduced pressure. The residue was redissolved in water $(0.5 \mathrm{ml})$ and subjected to electrophoresis, using Whatman No. 54 I paper on a Shandon high-voltage electrophoresis apparatus operated at $3 \mathrm{kV}$ for $2 \mathrm{~h}$. The solvent system used was formic acid-acetic acidwater (50:156:1794 by vol., $\mathrm{pH} 2 \cdot 0$ ). The paper was dried and the area corresponding to 2oxogluconate was eluted with distilled water. The resulting solution was taken to dryness by rotary evaporation and the electrophoretic separation repeated. The purity of the final product was greater than $99 \%$ as assessed by treating the radiochemical with various enzymes (gluconate kinase alone, or plus gluconate 6-phosphate dehydrogenase) and examining the incubation mixture for expected products (gluconate 6-phosphate and ribulose 5-phosphate, respectively) in the electrophoresis system described.

Analyses. Protein was estimated by the method of Gornall, Bardawill \& David (1949), with correction for the biuret-positive material in the glycylglycine buffer used to prepare bacterial extracts. Glucose was determined with a glucose oxidase kit (Boehringer Corp., London). 2-Oxogluconate was determined by the method of Lanning \& Cohen (195I). Glucose also reacts in this assay, though the sensitivity is 40-fold lower; its contribution was assessed from its known concentration in the sample, using a suitable calibration curve, and appropriate adjustment made in determining 2-oxogluconate. Gluconate was determined by coupling gluconate kinase with gluconate 6-phosphate dehydrogenase according to the Boehringer handbook. Other analyses were as performed by $\mathrm{Ng} \&$ Dawes (1973).

Chemicals. $\left[\mathrm{U}-{ }^{14} \mathrm{C}\right] \mathrm{glucose}$ and $\left[\mathrm{U}-{ }^{14} \mathrm{C}\right]$ gluconate were obtained from the Radiochemical Centre, Amersham, Buckinghamshire. Calcium 2-oxogluconate was obtained from Sigma and converted to its sodium salt by treatment with Dowex $50\left(\mathrm{H}^{+}\right.$form $)$followed by neutralization with IO M- $\mathrm{NaOH}$.

\section{RESULTS AND DISCUSSION}

A change of the limiting nutrient from ammonium to glucose had a profound effect on the transport systems (Table I). Thus the level of the glucose transport system increased some fivefold, whereas the levels of the transport of gluconate and 2-oxogluconate decreased respectively about seven- and fivefold. The induction of the glucose transport system that occurred on shifting from a nitrogen limitation to a glucose limitation probably reflected the decreased concentration of gluconate in the medium (Table 2), since we have shown, under conditions of nitrogen limitation, that gluconate produced by the extracellular activity of glucose dehydrogenase is a major factor in repressing glucose transport activity (Whiting 
Table 2. The steady state concentrations of ammonium, glucose, gluconate and 2-oxogluconate determined during glucose- or nitrogen-limited growth of $P$. aeruginosa PAOI

\begin{tabular}{lcccc} 
Limiting & \multicolumn{4}{c}{ Residual concentration* (mM) } \\
nutrient & Ammonium & Glucose & Gluconate & 2-Oxogluconate \\
Ammonium & ND & 8.7 & 2.2 & 4.7 \\
& ND & 8.8 & 2.9 & 4.9 \\
Glucose & 13.2 & 0.1 & 0.1 & 0.4 \\
& 13.4 & 0.1 & 0.3 & 0.2
\end{tabular}

ND, Not detected.

* The two values quoted are the means of duplicate determinations performed on two separate culture samples from each steady state.

et al., 1976). The fall in the levels of the gluconate and 2-oxogluconate transport systems can be attributed to the reduction in concentration of gluconate and 2-oxogluconate respectively, since we have also demonstrated (Whiting et al., 1976) that glucose must be converted to these substrates for the induction of their transport systems. The relatively high $K_{m}$ values (both about I $\mathrm{mm}$ ) of the enzymes of the extracellular pathway (Midgley \& Dawes, 1973; Roberts et al., 1973) compared with the $K_{m}$ for glucose transport $(8 \mu \mathrm{M})$, plus the observed induction of the glucose transport system, presumably results in a much diminished flux of carbon through the extracellular pathway under conditions of glucose limitation when the residual glucose concentration is approximately o. $\mathrm{I} \mathrm{mM}$.

The capacity of the organism to transport 2-oxogluconate was large compared with the other two transport systems when grown under nitrogen limitation, conditions which produced approximately $5 \mathrm{~mm}-2$-oxogluconate in the medium. Even higher activities of this transport system have been detected in the organism grown in batch culture on gluconate when, by a conventional Lineweaver-Burk plot, the $K_{m}$ and $V_{\text {max. }}$ for 2-oxogluconate uptake were determined as $0.9 \mathrm{~mm}$ and $118 \mu \mathrm{mol} / \mathrm{min} / \mathrm{g}$ dry wt respectively. The relatively high activity of this transport system, under conditions of nitrogen limitation, may reflect the excess activity of the extracellular pathway enzymes [approximately ro- to 20-fold (Midgley \& Dawes, 1973; Roberts et al., 1973)], compared with the activities of the glucose and gluconate transport systems.

The switch from the extracellular pathway to the intracellular pathway when the organism is subjected to a change from a nitrogen to a glucose limitation is also reflected by the levels of the enzymes of glucose catabolism. Glucose limitation resulted in a marked decrease in the levels of gluconate dehydrogenase, glucose dehydrogenase, gluconate kinase, and the enzymes of 2-oxogluconate catabolism assayed as a combined activity (Table 3 ). In contrast, the enzymes which initiate the intracellular phosphorylative metabolism, namely hexokinase and glucose 6-phosphate dehydrogenase, were substantially increased.

As previously noted, the extracellular and intracellular routes converge on gluconate 6phosphate for entry to the Entner-Doudoroff pathway and the enzymes of this latter sequence are not significantly affected by the switch from nitrogen to glucose limitation. However, the activity of one of the enzymes of the tricarboxylic acid cycle, isocitrate dehydrogenase, falls by two-thirds.

It is clear, therefore, that $P$. aeruginosa responds to a limited supply of carbon by diverting its metabolism of glucose substantially from the extracellular direct oxidative pathway to the intracellular phosphorylative route. Despite the high steady state concentration of 2-oxogluconate produced under conditions of nitrogen limitation in the chemostat, and there- 


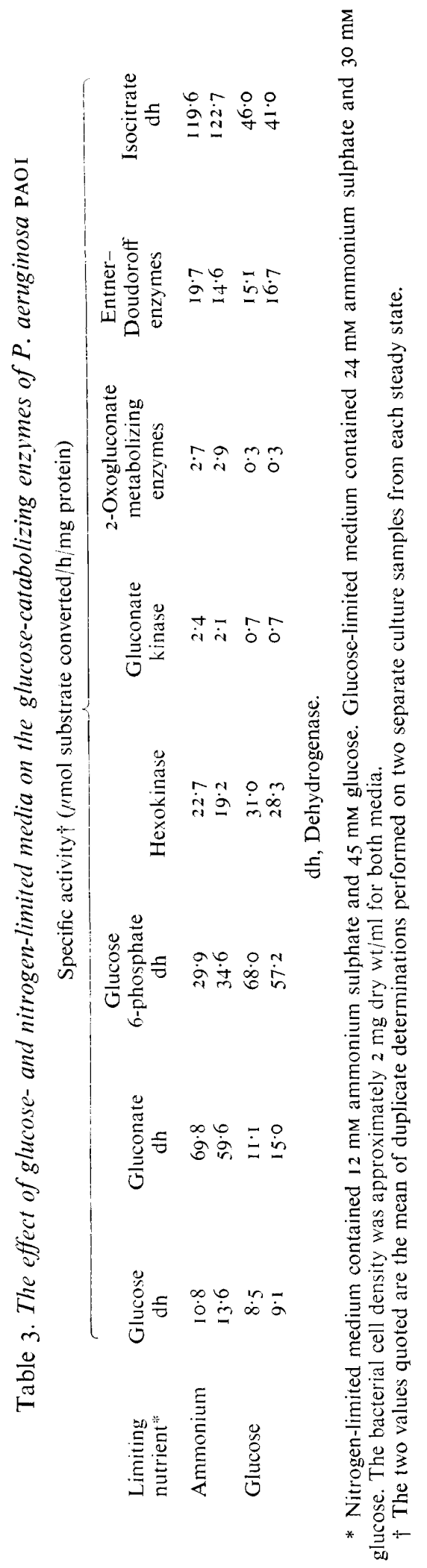


fore lost in the outflow, there does not appear to to be a large wastage of glucose carbon since the steady state rate of glucose utilization under conditions of nitrogen-limitation is only slightly greater $(6.8$ against $6.2 \mathrm{mmol} / \mathrm{h} / \mathrm{g}$ dry $\mathrm{wt})$ than the rate under conditions of carbon limitation.

Our findings thus offer some support for the suggestion that the persistence, during the course of evolution, of the extracellular pathway of glucose metabolism in P. aeruginosa, and in Pseudomonas fluorescens (Eisenberg et al., 1974), has permitted these organisms, in their natural habitat, to sequester glucose as gluconate and 2-oxogluconate, compounds which are not so readily utilized by various other micro-organisms that may effectively compete for the available glucose. Thus, when the glucose supply is restricted, $P$. aeruginosa responds by adjusting its metabolism so that the available carbon source is taken up as rapidly as possible. Pseudomonas aeruginosa therefore affords an excellent example of microbial response to a low-nutrient environment, as discussed by Tempest (1976) for Klebsiella aerogenes in respect of both ammonium and glycerol uptake.

We thank the Science Research Council for the award of a Research Studentship to P.H.W.

\section{REFERENCES}

Claridge, C. A. \& Werkman, C. H. (1953). Formation of 2-ketogluconate from glucose by a cell-free preparation of Pseudomonas aeruginosa. Archives of Biochemistry and Biophysics 47, 99-106.

Claridge, C. A. \& Werkman, C. H. (1954). Evidence for alternate pathways for the oxidation of glucose by Pseudomonas aeruginosa. Journal of Bacteriology 68, 77-79.

Dawes, E. A., Midgley, M. \& Whiting, P. H. (1976). The control of transport systems for glucose, gluconate and 2-oxogluconate, and of glucose metabolism in Pseudomonas aeruginosa. In Proceedings of the Sixth International Symposium and Study Group on the Continuous Culture of Micro-organisms, Oxford. Chichester: Ellis-Hall.

Eisenberg, R. C., Butters, S. J., Quay, S. C. \& Friedman, S. B. (1974). Glucose uptake and phosphorylation in Pseudomonas fluorescens. Journal of Bacteriology 120, I47-1 53.

Gornall, A. G., Bardawill, C. J. \& David, M. M. (1949). Determination of serum proteins by means of the biuret reaction. Journal of Biological Chemistry $\mathbf{1 7 7}, 75 \mathrm{I}-766$.

Hamlin, B. T., NG, F. M-W. \& Dawes, E. A. (1967). Regulation of enzymes of glucose metabolism in Pseudomonas aeruginosa by citrate. In Microbial Physiology and Continuous Culture, pp. $21 \mathrm{I}-23 \mathrm{I}$. London: H.M.S.O.

LAnning, M. C. \& Cohen, S. S. (195I). The detection and estimation of 2-ketohexonic acid. Journal of Biological Chemistry 189, 109-114.

Midgley, M. \& Dawes, E. A. (1973). The regulation of transport of glucose and methyl $\alpha$-glucoside in Pseudomonas aeruginosa. Biochemical Journal $\mathbf{1 3 2}$, 4 [ I-154.

NG, F. M-W. \& DAwEs, E. A. (1973). Chemostat studies on the regulation of glucose metabolism in Pseudomonas aeruginosa by citrate. Biochemical Journal 132, I29-140.

Roberts, B. K., Midgley, M. \& Dawes, E. A. (1973). The metabolism of 2-oxogluconate by Pseudomonas aeruginosa. Journal of General Microbiology 78, 319-329.

Stokes, F. N. \& Campbell, J. J. R. (195I). The oxidation of glucose and gluconic acid by dried cells of Pseudomonas aeruginosa. Archives of Biochemistry and Biophysics 30, $121-125$.

Tempest, D. W. (1976). The application of continuous culture to studies of microbial adaptation to lownutrient environments. In Proceedings of the Sixth International Symposium and Study Group on Continuous Culture, Oxford. Chichester: Ellis-Hall.

Wang, C. H., Stern, I. J. \& Gilmour, C. M. (1959). The catabolism of glucose and gluconate in Pseudomonas species. Archives of Biochemistry and Biophysics 8r, 489-492.

Whiting, P. H., Midgley, M. \& Dawes, E. A. (I976). The regulation of transport of glucose, gluconate and 2-oxogluconate, and of glucose catabolism, in Pseudomonas aeruginosa. Biochemical Journal (in the Press). 